\title{
Stokes solitons in optical microcavities
}

\author{
Qi-Fan Yang ${ }^{\dagger}, \mathrm{Xu} \mathrm{Yi}^{\dagger}$, Ki Youl Yang and Kerry Vahala*
}

Solitons are wave packets that resist dispersion through a self-induced potential well. They are studied in many fields, but are especially well known in optics on account of the relative ease of their formation and control in optical fibre waveguides $^{1,2}$. Besides their many interesting properties, solitons are important to optical continuum generation ${ }^{3}$, in mode-locked lasers ${ }^{4,5}$, and have been considered as a natural way to convey data over great distances ${ }^{6}$. Recently, solitons have been realized in microcavities ${ }^{7}$, thereby bringing the power of microfabrication methods to future applications. This work reports a soliton not previously observed in optical systems, the Stokes soliton. The Stokes soliton forms and regenerates by optimizing its Raman interaction in space and time within an optical potential well shared with another soliton. The Stokes and the initial soliton belong to distinct transverse mode families and benefit from a form of soliton trapping that is new to microcavities and soliton lasers in general. The discovery of a new optical soliton can impact work in other areas of photonics, including nonlinear optics and spectroscopy.

Solitons result from a balance of wave dispersion with a nonlinearity. In optics, temporal solitons are readily formed in optical fibre waveguides ${ }^{1-3,6}$ and laser resonators ${ }^{4,5}$, and have recently been observed in dielectric microcavities ${ }^{7}$. In each of these cases nonlinear compensation of group velocity dispersion is provided by the Kerr effect (nonlinear refractive index). Besides the Kerr nonlinearity, a secondary effect associated with soliton propagation is the so-called soliton self-frequency shift caused by Raman interaction, which induces a continuously increasing redshift with propagation in a waveguide $^{3}$ or a fixed shift of the soliton spectrum in cavities ${ }^{8,9}$. More generally, the Raman interaction can produce optical amplification and laser action of waves redshifted relative to a strong pumping wave or puls $\mathrm{e}^{10-12}$. This work reports a new Raman-related effect, soliton generation through Kerr-effect trapping and Raman amplification created by the presence of a first temporal soliton. Because the new soliton is spectrally redshifted relative to the initial soliton we call it a Stokes soliton. It is observed in a silica microcavity and obeys a threshold condition resulting from an optimal balancing of Raman gain with cavity loss when the soliton pulses overlap in space and time. Also, the repetition frequency of both the initial and the Stokes soliton are locked by the Kerr nonlinearity.

In this work the resonator is a circular-shaped whispering gallery microcavity and the first temporal soliton will be referred to as the primary soliton. The primary soliton here is a dissipative Kerr soliton (DKS) $)^{7,13,14}$. Consistent with its formation, the primary soliton creates a spatially varying refractive index via the Kerr nonlinearity that serves as an effective potential well. It travels with the soliton and counteracts optical dispersion. Moreover, on account of the Raman interaction, the primary soliton creates local Raman amplification that also propagates with the primary soliton.
These propagating index and gain profiles are depicted in Fig. 1a. The primary soliton is composed of many longitudinal modes belonging to one of the transverse mode families of the cavity. These phase-locked modes form a frequency comb. $\Delta v_{\mathrm{P}}$ will denote the longitudinal mode separation or free spectral range (FSR) for longitudinal modes near the spectral centre of the primary soliton. $\Delta v_{\mathrm{P}}$ also gives the approximate round-trip rate of the primary soliton around the cavity ( $T_{\mathrm{RT}}=\Delta v_{\mathrm{P}}^{-1}$ is the round-trip time).

Consider another transverse mode family besides the one that forms the primary soliton. Suppose that some group of longitudinal modes in this family satisfies two conditions: Condition 1 is that they lie within the Raman gain spectrum created by the primary soliton; Condition 2 is that they feature an FSR that is close in value to that of the primary soliton $\left(\Delta v_{\mathrm{P}}\right)$. Any noise in these longitudinal modes will be amplified by Raman gain provided by the primary soliton. If the round-trip amplification of a resulting waveform created by a superposition of these modes is sufficient to overcome round-trip optical loss, then oscillation threshold is possible. The threshold will be lowest (Raman gain maximal) if the modes of the second family phase lock to form a pulse overlapping in both space and time with the primary soliton. This overlap is possible since the round-trip time of the primary soliton and the new pulse are closely matched, that is, Condition 2 above is satisfied. Also, the potential well created by the primary soliton can be shared with the new optical pulse. This latter nonlinear coupling of the primary soliton with the new, Stokes soliton pulse results from Kerrmediated cross-phase modulation and locks the round-trip rates of the two solitons (that is, their soliton pulse repetition frequencies are locked).

The generation of a fundamental soliton by another fundamental soliton in this way is new and also represents a form of mode locking of a soliton laser. It differs from mechanisms such as soliton fission which also result in the creation of one of more fundamental solitons ${ }^{3}$. Specifically, soliton fission involves a higher-order soliton breaking into multiple fundamental solitons; nor is it a regenerative process. Coexisting and switching dissipative solitons have been observed $^{15}$ and modelled ${ }^{15,16}$ in an erbium-doped mode-locked fibre laser. Also, mode locking that results in stable multi-colour solitons has been observed in an ytterbium laser by using a fiber re-injection loop to in effect synchronously pump the system via the Raman process ${ }^{17}$. However, in the present case, the Stokes soliton relies upon the primary soliton for its existence by leveraging spatial-temporal overlap for both Kerr-effect trapping and optical amplification. Raman gain produced for the Stokes soliton by the primary soliton is also distinctly different from the Raman self-frequency shift ${ }^{18,19}$, which is an effect of the Raman interaction on the primary soliton $^{8,9,20}$. Also, this new Raman mechanism is responsible for oscillation of the Stokes soliton at a well-defined threshold. Raman self-shift is, on the other hand, not a thresholding process. Finally, concerning the trapping phenomenon that accompanies the Stokes 
$\mathbf{a}$

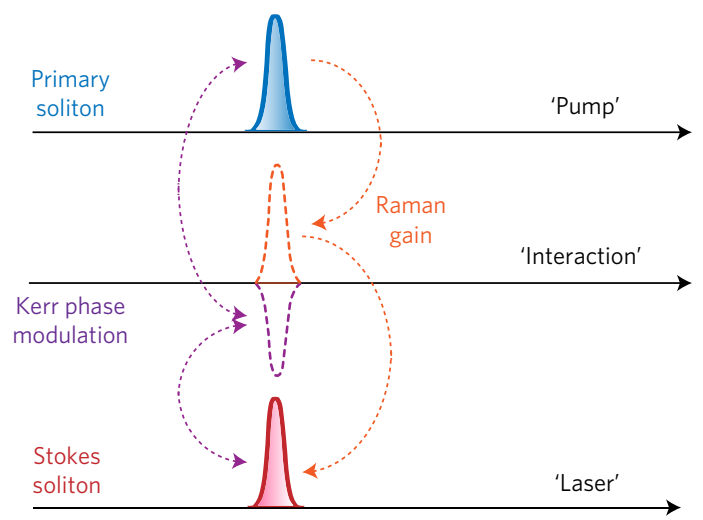

b

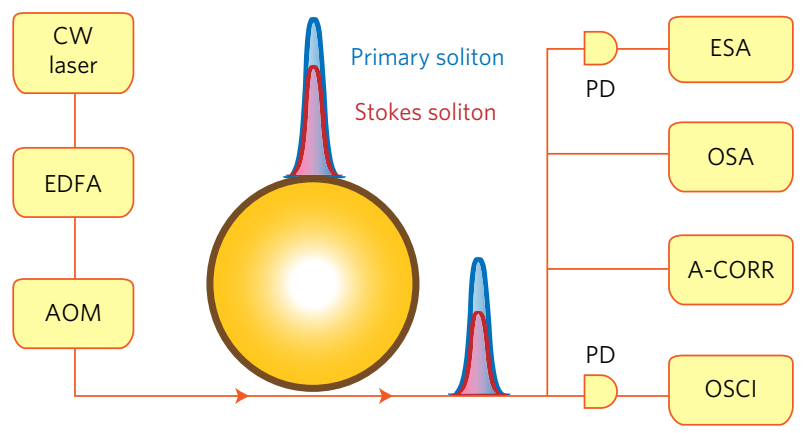

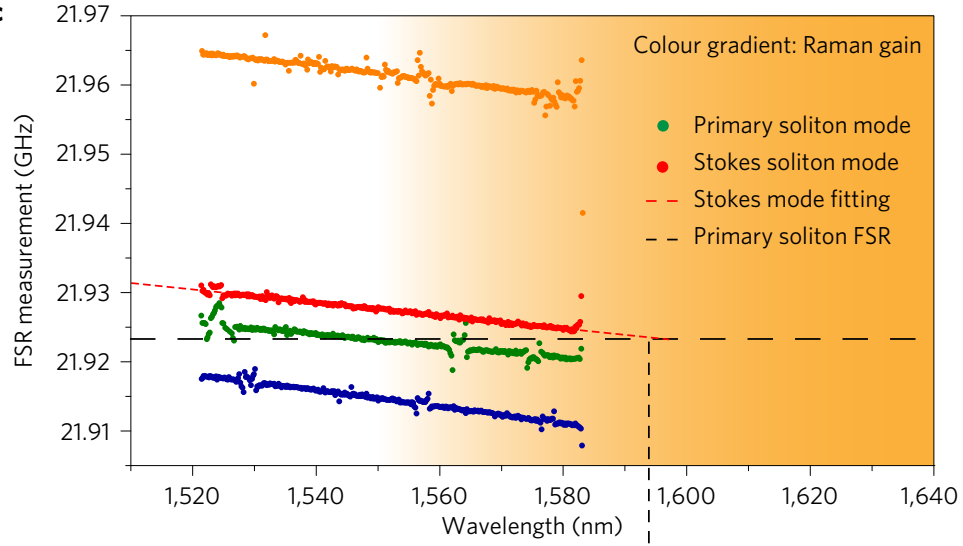

d

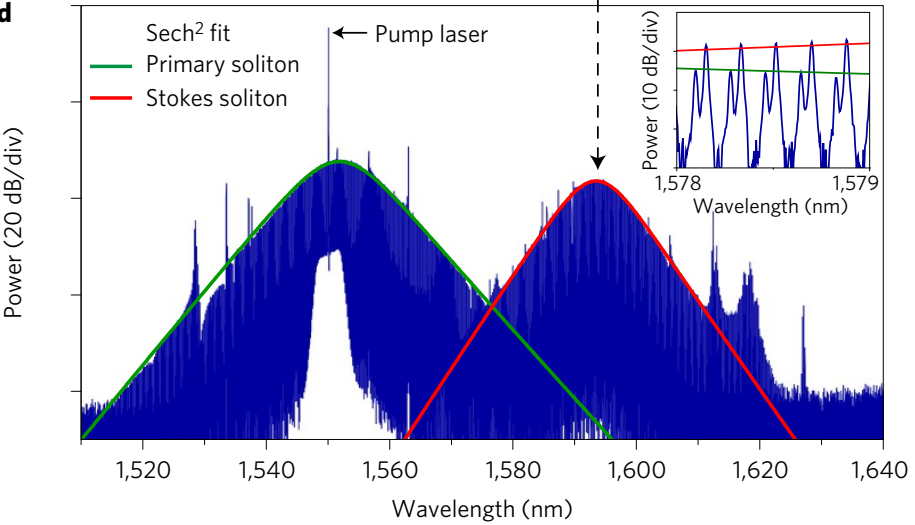

Figure 1 | Experimental set-up and description of Stokes soliton generation. a, The Stokes soliton (red) maximizes Raman gain by overlapping in time and space with the primary soliton (blue). It is also trapped by the Kerr-induced effective optical well created by the primary soliton. $\mathbf{b}$, The microcavity (shown as a ring) is pumped with a tunable, continous-wave (CW) fibre laser amplified by an erbium-doped fibre amplifier (EDFA). An acousto-optic modulator (AOM) is used to control the pump power. The output soliton power is detected with a photodiode (PD) and monitored on an oscilloscope (OSCI). Wavelength division multiplexers (not shown) split the 1,550 nm band primary soliton and 1,600 nm band Stokes soliton so that their powers can be monitored separately on the oscilloscope. An optical spectrum analyzer (OSA), auto-correlator (A-CORR) and electrical spectrum analyzer (ESA) also monitor the output. In certain measurements (A-CORR and ESA), a fibre Bragg filter was used to remove the pump field from the soliton spectrum. c, Free spectral range (FSR) versus wavelength measured for four mode families in a $3 \mathrm{~mm}$ disk resonator. The mode families for the primary and Stokes soliton are shown in green and red, respectively. The FSR at the spectral centre of the primary soliton is shown as a dashed horizontal line. Extrapolation of the Stokes soliton data (red) to longer wavelengths gives the FSR-matching wavelength where the Stokes soliton forms. The background coloration gives the approximate wavelength range of the Raman gain spectrum. d, Measured primary and Stokes soliton spectra. The Stokes soliton spectral centre closely matches the prediction in $\mathbf{c}$. Sech ${ }^{2}$ envelopes are shown on each spectrum. The primary soliton spectrum features a small Raman self-frequency shift.

soliton formation, the trapping of temporal solitons belonging to distinct polarization states was proposed in the late $1980 \mathrm{~s}^{21}$ and was observed in optical fibre $e^{22,23}$ and later in fibre lasers ${ }^{24}$. However, trapping of temporal solitons belonging to distinct transverse mode families, as observed here, was proposed even earlier ${ }^{25,26}$, but has only recently been observed in graded-index fibre waveguides ${ }^{27}$. Trapping of solitons in microcavities has never been reported, nor has the spatial-temporal formation of a soliton through the Raman process. The observation, measurement and modelling of Stokes solitons is now presented.

The experimental set-up is shown in Fig. 1b. The microcavities are about $3 \mathrm{~mm}$ in diameter, fabricated from silica on silicon and have an unloaded optical $Q$ factor of 250 million $^{28}$. The cavity is pumped by a continuous-wave fibre laser to induce a primary soliton which is a DKS with a repetition frequency of $22 \mathrm{GHz}$ and a pulse width that could be controlled to lie within the range of 100-200 fs. The requirements for DKS generation include a mode family that features anomalous dispersion. Other requirements as well as control of DKS properties are described elsewhere ${ }^{7,20}$. Measurement of the FSR versus wavelength of four spatial mode families in a single resonator is presented in Fig. 1c. The measurement is performed by scanning a tunable external cavity diode laser
(ECDL) through the spectral locations of optical resonances from $1,520 \mathrm{~nm}$ to $1,580 \mathrm{~nm}$. The resonances appear as minima in the optical power transmitted past the microcavity, and the location of these resonances is calibrated using a fibre-based Mach-Zehnder interferometer. The resulting data provide the dispersion in the FSR of cavity modes versus the wavelength, and readily enable the identification transverse mode families. Other details on this method are described elsewhere ${ }^{20}$. The green data plotted in Fig. 1c correspond to the primary soliton-forming mode family and the FSR of the pumping mode for that soliton is indicated by the horizontal dashed line. The measured spectrum for the primary soliton is shown in Fig. 1d. In the spectrum the pump spectral line (near $1,550 \mathrm{~nm}$ ) is indicated as well as the sech $^{2}$ envelope for the soliton (green curve) $)^{7,20}$. Returning to Fig. 1c, the dispersion for another spatial mode family (red data) is extrapolated to longer wavelengths and crosses the dashed line near $1,593 \mathrm{~nm}$. At this wavelength the FSR of the red mode family closely matches that of the primary soliton. Moreover, this wavelength also lies within the Raman gain spectrum produced by the primary soliton (amber region in Fig. 1c gives the Raman gain band). As a result, Conditions 1 and 2 above are satisfied at this wavelength for generation of a Stokes soliton. The corresponding measured Stokes soliton is shown 


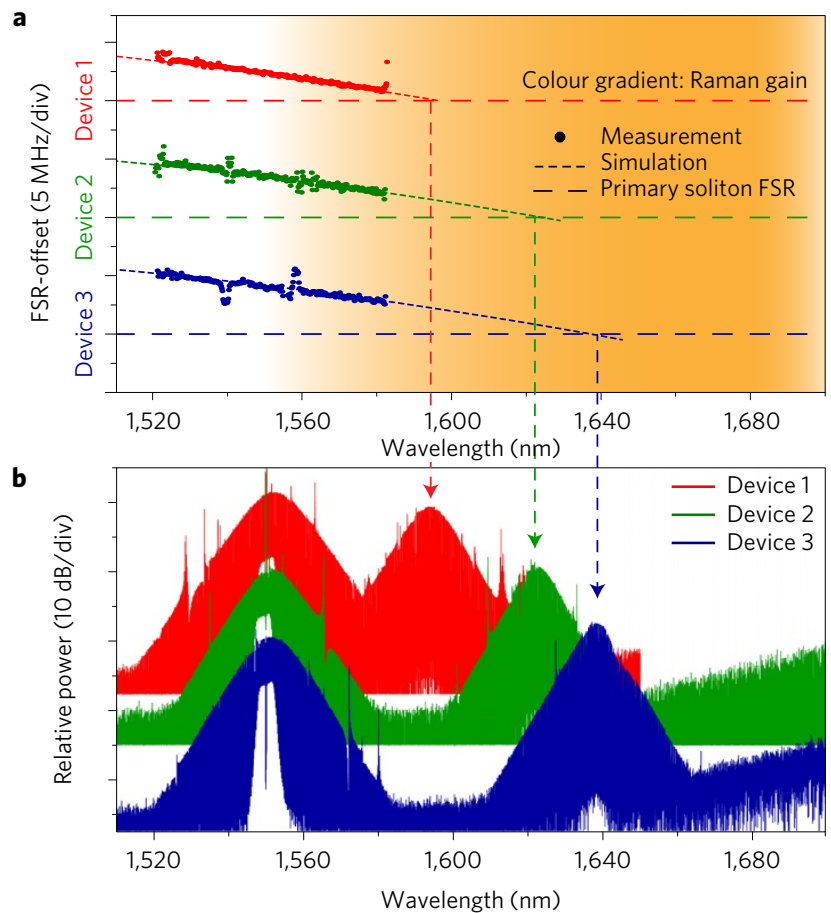

Figure 2 | Observation of Stokes solitons in multiple devices. a, Dispersion spectra (see Fig. 1c) for the Stokes-soliton-forming mode family measured in three devices (the upper spectrum is the device from Fig. 1). Other mode families have been omitted in the plots for clarity. The horizontal dashed lines give the repetition frequency of the primary soliton in each device. Extrapolation of the dispersion data attained by simulation is provided to graphically locate the predicted Stokes soliton wavelength. b. The measured primary and Stokes soliton spectra corresponding to the devices in a. The spectral locations of the Stokes solitons agree well with the graphical predictions.

in Fig. 1d. Its spectral maximum occurs at a wavelength that agrees well with the prediction based on the FSR measurement in Fig. 1c. As an aside, the individual spectral lines of the primary and the Stokes solitons are spaced by approximately $22 \mathrm{GHz}$ and resolved comb lines in the overlapping spectral region between the solitons are provided in the inset to Fig. 1d.

These results were readily reproduced in other devices. Indeed, the FSR-matching wavelength (that is, Condition 2) could be controlled by adjusting the geometry of the resonator. Figure 2a shows the FSR dispersion measurements and Fig. $2 \mathrm{~b}$ shows the corresponding primary and Stokes soliton spectra that are measured using two other devices (green and blue spectra). For clarity, only the Stokes-soliton-forming mode family is presented in Fig. 2a (including the data from Fig. 1c, red spectra, for comparison). The FSR of the primary soliton is indicated by the horizontal dashed lines in Fig. 2a. The FSR-matching wavelengths are indicated by extrapolation of the dispersion data using simulation ${ }^{29}$ and agree well with the location of the measured Stokes soliton spectral maximum in Fig. 2b.

To confirm the temporal pulse nature of the Stokes soliton the frequency-resolved optical gate (FROG) method was used to record the correlation traces in Fig. 3a. Optical filtering was applied to isolate the primary and Stokes soliton pulse streams. Also, before FROG measurement, the primary soliton stream was amplified by an erbium-doped fibre amplifier (EDFA) and the Stokes soliton was amplified using a semiconductor optical amplifier (SOA). The data confirm that both streams consist of pulses. As an aside, the signalto-noise level for the Stokes soliton measurement was limited by the saturation power of the SOA.
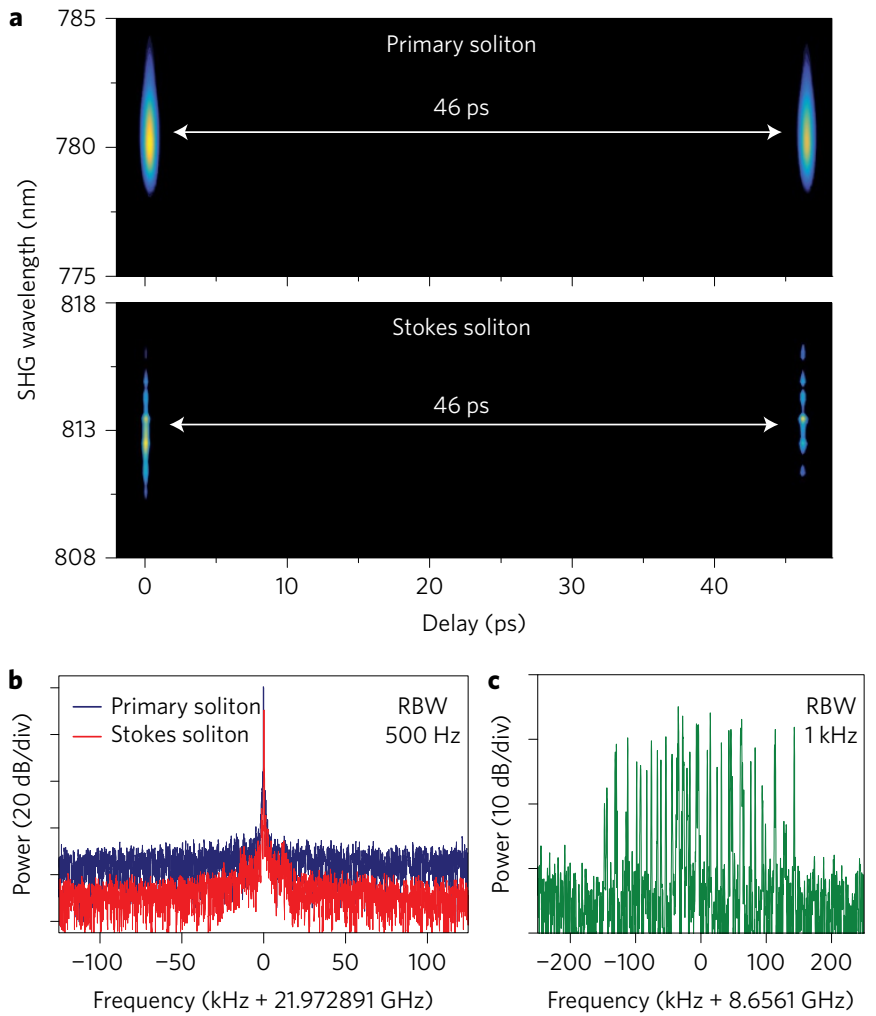

Figure 3 | Soliton pulse and frequency measurements.

a, Frequency-resolved optical gating (FROG) traces of the primary and Stokes solitons. The primary soliton is amplified to $500 \mathrm{~mW}$ by an EDFA before coupling into the FROG set-up, while the Stokes soliton is amplified to $10 \mathrm{~mW}$ by two cascaded semiconductor optical amplifiers with gain centred around $1,620 \mathrm{~nm}$. The period of the primary and Stokes solitons are 46 ps, the cavity round-trip time. b. Electrical spectra of the detected primary soliton pulse stream (blue) and the Stokes pulse stream (red). c, Beatnote between neighbouring comb lines of the primary and Stokes solitons for a device like that in Fig. 1. The beatnote is much noisier than the repetition frequency in $\mathbf{b}$.

Photodetection of the isolated primary and Stokes soliton pulse streams was also possible. The measured electrical spectrum for each pulse stream is overlaid in Fig. 3b. The spectra are observed to align in frequency. As further confirmation of this frequency alignment, the optical pulse streams were simultaneously detected (no filtering to isolate the streams) and the measured electrical spectrum featured a single spectral peak at a resolution of $500 \mathrm{~Hz}$. These results are expected on account of optical trapping of the Stokes soliton by the primary soliton which causes the line spacing (repetition rate) of the Stokes soliton and the primary soliton to match.

At the same time, the absolute optical frequency of the two solitons is not expected to be locked since the optical potential well depends upon the intensity of the primary soliton. To test this, the relative optical frequency stability of the two soliton streams was measured by using a resonator that featured spectrally overlapping primary and Stokes solitons such as in Fig. 1d. A high-resolution optical scan from 1,578 to $1,579 \mathrm{~nm}$ of this overlap region is presented as the inset in Fig. 1d and confirms that the underlying soliton lines are distinct. The beatnote between neighbouring spectral lines of the primary and Stokes solitons shows large frequency variations (see Fig. 3c) in comparison with the repetition frequency of each soliton (Fig. 3b). Synchronization of the soliton repetition rates, but not of the absolute optical frequencies, is thus inferred from these measurements. 

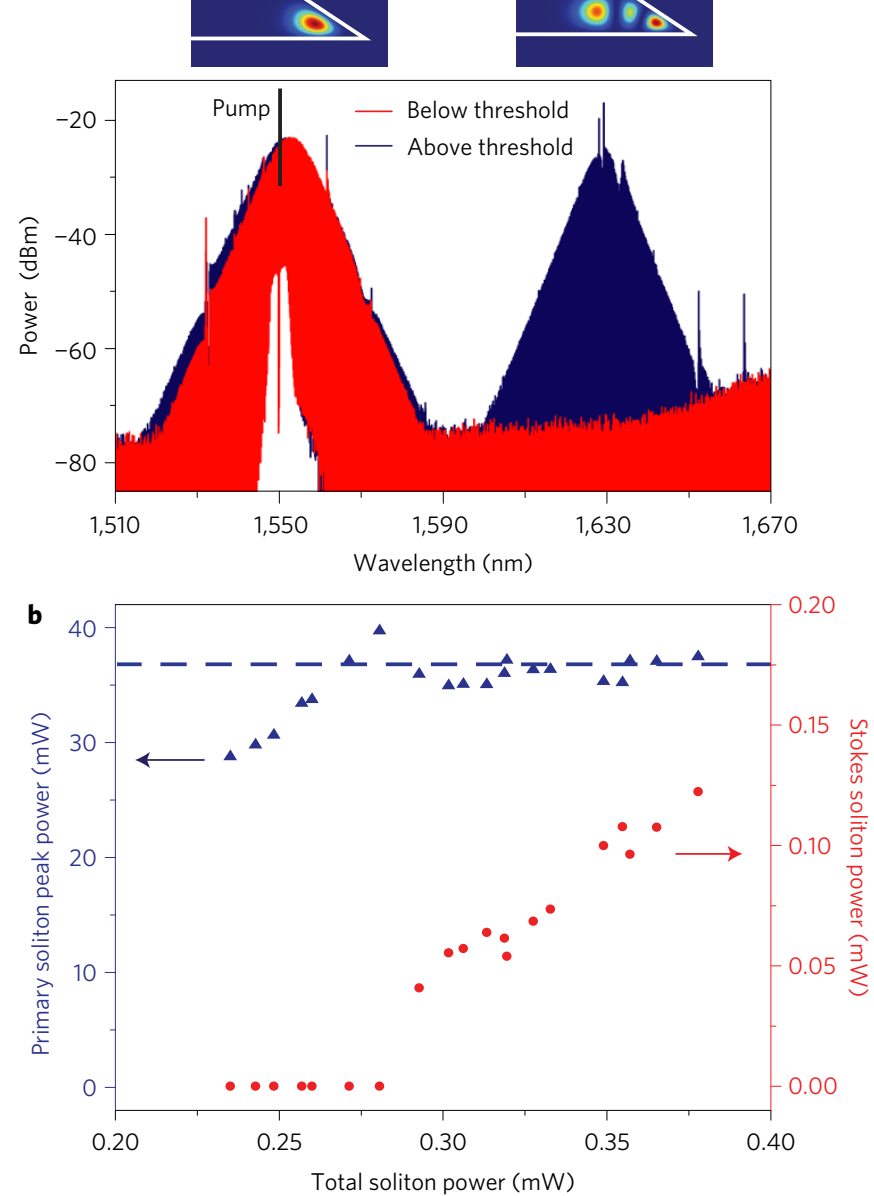

Figure 4 | Stokes soliton spectra, power and threshold measurements.

a, Soliton spectra are plotted below and above threshold. The upper insets show the spatial mode families associated with the primary and Stokes solitons. b, Measurement of Stokes soliton power and primary soliton peak power versus total soliton power. The primary soliton peak power (blue) versus total power experiences threshold clamping at the onset of Stokes soliton oscillation. The theoretical threshold peak power from equation (1) is also shown for comparison as the horizontal blue dashed line.

The thresholding nature of Stokes soliton formation is studied in Fig. 4. The spectra in Fig. 4a show the primary soliton and the corresponding Stokes soliton spectra for pumping levels below and above threshold. The transverse spatial mode profiles on which these solitons are formed are also provided and have been inferred by numerical fitting to the dispersion data. In Fig. $4 \mathrm{~b}$ power data are provided showing the primary soliton peak power and the Stokes soliton power plotted versus the total soliton power. To measure this data, the power in the primary soliton was varied using the locking method described elsewhere ${ }^{30}$. Also, the transmitted pump spectral line was filtered out using a fibre Bragg filter. Power was measured by summing the power of the respective comb lines for the primary and Stokes soliton as measured on the optical spectrum analyzer. Consistent with the thresholding nature of the process, the Stokes soliton power could be increased beyond the primary soliton power (for example, see Supplementary Fig. 1 in Supplementary Information).

A threshold-induced gain-clamping condition is imposed on the primary soliton peak power (which functions as a pump for the Stokes soliton). The clamping of peak primary soliton power is readily observable in Fig. 4b. This threshold condition is studied in detail in the Methods, where the threshold peak power is shown to be given by the following expression,

$$
P_{\mathrm{th}}=\frac{\kappa_{\mathrm{p}}^{\mathrm{ext}} \kappa_{\mathrm{s}}}{2 R}\left(1+\frac{1}{2 \gamma}\right)
$$

where $P_{\text {th }}$ is the threshold peak output power of the primary soliton and other quantities are defined in the Methods. Equation (1) is derived assuming a weak Stokes soliton power relative to the primary soliton, which is an excellent assumption near threshold. The threshold peak power predicted by equation (1) is plotted as the horizontal dashed line in Fig. $4 \mathrm{~b}$ for comparison to measurements. The values used in this calculation are provided in the Methods and are calculated or measured.

The Stokes soliton is only the second type of soliton to be observed in microcavities (beyond dissipative Kerr solitons ${ }^{7}$ ) and also represents the first time soliton trapping has been observed in any microcavity. It also represents the first observation of trapping by solitons in different transverse modes in a laser. From a practical viewpoint, the Stokes and primary solitons overlap in space and time, and have a frequency separation that can be engineered to fall within the mid-infrared range. As a result, this soliton system is potentially interesting for midinfrared generation by way of difference frequency generation. Not all devices are observed to produce Stokes solitons. However, dispersion engineering techniques are being advanced ${ }^{29}$ and should enable control of both observation of the Stokes soliton as well as its placement in the optical spectrum. Indeed, the spectral placement of Stokes solitons in Fig. 2b is largely the result of microcavity diameter control to shift the FSR crossing point. Also, engineering of dispersion could permit a Stokes soliton to form within the same mode family as the primary soliton. The specific implementation described here uses a compact microresonator on a silicon wafer, which also suggests that monolithic integration will be possible. In an appropriately phase-matched multimode waveguide (optical fibre or monolithic) it should also be possible to observe non-cavitybased Stokes solitons using a pulse seed.

\section{Methods}

Methods, including statements of data availability and any associated accession codes and references, are available in the online version of this paper.

\section{Received 10 January 2016; accepted 3 August 2016;} published online 5 September 2016

\section{References}

1. Hasegawa, A. \& Tappert, F. Transmission of stationary nonlinear optical pulses in dispersive dielectric fibers. i. anomalous dispersion. Appl. Phys. Lett. 23, 142-144 (1973).

2. Mollenauer, L. F., Stolen, R. H. \& Gordon, J. P. Experimental observation of picosecond pulse narrowing and solitons in optical fibers. Phys. Rev. Lett. 45, 1095-1098 (1980).

3. Dudley, J. M., Genty, G. \& Coen, S. Supercontinuum generation in photonic crystal fiber. Rev. Mod. Phys. 78, 1135-1184 (2006).

4. Haus, H. A. et al. Mode-locking of lasers. IEEE J. Sel. Top. Quantum Electron. 6, 1173-1185 (2000).

5. Cundiff, S. Dissipative Solitons 183-206 (Springer, 2005).

6. Haus, H. A. \& Wong, W. S. Solitons in optical communications. Rev. Mod. Phys. 68, 423-444 (1996).

7. Herr, T. et al. Temporal solitons in optical microresonators. Nat. Photon. 8, 145-152 (2014).

8. Karpov, M. et al. Raman self-frequency shift of dissipative Kerr solitons in an optical microresonator. Phys. Rev. Lett. 116, 103902 (2016).

9. Yi, X., Yang, Q.-F., Yang, K. Y. \& Vahala, K. Theory and measurement of the soliton self-frequency shift and efficiency in optical microcavities. Opt. Lett. 41, 3419-3422 (2016)

10. Islam, M. N. Raman amplifiers for telecommunications. IEEE J. Sel. Top. Quantum Electron. 8, 548-559 (2002). 
11. Spillane, S., Kippenberg, T. \& Vahala, K. Ultralow-threshold Raman laser using a spherical dielectric microcavity. Nature 415, 621-623 (2002).

12. Headley, C. \& Agrawal, G. P. Unified description of ultrafast stimulated Raman scattering in optical fibers. J. Opt. Soc. Am. B 13, 2170-2177 (1996).

13. Akhmediev, N. \& Ankiewicz, A. Dissipative Solitons: From Optics to Biology and Medicine (Springer, 2008).

14. Leo, F. et al. Temporal cavity solitons in one-dimensional Kerr media as bits in an all-optical buffer. Nat. Photon. 4, 471-476 (2010).

15. Bao, C., Chang, W., Yang, C., Akhmediev, N. \& Cundiff, S. T. Observation of coexisting dissipative solitons in a mode-locked fiber laser. Phys. Rev. Lett. 115, 253903 (2015).

16. Soto-Crespo, J. M. \& Akhmediev, N. Composite solitons and two-pulse generation in passively mode-locked lasers modeled by the complex quintic Swift-Hohenberg equation. Phys. Rev. E 66, 066610 (2002).

17. Babin, S. A. et al. Multicolour nonlinearly bound chirped dissipative solitons. Nat. Commun. 5, 4653 (2014).

18. Mitschke, F. M. \& Mollenauer, L. F. Discovery of the soliton self-frequency shift. Opt. Lett. 11, 659-661 (1986).

19. Gordon, J. P. Theory of the soliton self-frequency shift. Opt. Lett. 11, 662-664 (1986)

20. Yi, X., Yang, Q.-F., Yang, K. Y., Suh, M.-G. \& Vahala, K. Soliton frequency comb at microwave rates in a high-Q silica microresonator. Optica 2, 1078-1085 (2015).

21. Menyuk, C. R. Stability of solitons in birefringent optical fibers. i: equal propagation amplitudes. Opt. Lett. 12, 614-616 (1987).

22. Islam, M., Poole, C. \& Gordon, J. Soliton trapping in birefringent optical fibers. Opt. Lett. 14, 1011-1013 (1989).

23. Cundiff, S. et al. Observation of polarization-locked vector solitons in an optical fiber. Phys. Rev. Lett. 82, 3988-3991 (1999).

24. Collings, B. C. et al. Polarization-locked temporal vector solitons in a fiber laser: experiment. J. Opt. Soc. Am. B 17, 354-365 (2000).

25. Hasegawa, A. Self-confinement of multimode optical pulse in a glass fiber. Opt. Lett. 5, 416-417 (1980).
26. Crosignani, B. \& Di Porto, P. Soliton propagation in multimode optical fibers. Opt. Lett. 6, 329-330 (1981)

27. Renninger, W. H. \& Wise, F. W. Optical solitons in graded-index multimode fibres. Nat. Commun. 4, 1719 (2013)

28. Lee, H. et al. Chemically etched ultrahigh-Q wedge-resonator on a silicon chip. Nat. Photon. 6, 369-373 (2012)

29. Yang, K. Y. et al. Broadband dispersion-engineered microresonator on-a-chip Nat. Photon. 10, 316-320 (2016)

30. Yi, X., Yang, Q.-F., Yang, K. Y. \& Vahala, K. Active capture and stabilization of temporal solitons in microresonators. Opt. Lett. 41, 2037-2040 (2016).

\section{Acknowledgements}

The authors thank S. Cundiff at the University of Michigan for helpful comments on this manuscript. The authors gratefully acknowledge the Defense Advanced Research

Projects Agency under the PULSE Program, NASA, the Kavli Nanoscience Institute and the Institute for Quantum Information and Matter, an NSF Physics Frontiers Center with support of the Gordon and Betty Moore Foundation.

\section{Author contributions}

Experiments were conceived by all co-authors. Analysis of results was conducted by al co-authors. Q.-F.Y. and X.Y. performed measurements. K.Y.Y. fabricated devices. Al authors participated in writing the manuscript.

\section{Additional information}

Supplementary information is available in the online version of the paper. Reprints and permissions information is available online at www.nature.com/reprints.

Correspondence and requests for materials should be addressed to K.V.

\section{Competing financial interests}

The authors declare no competing financial interests. 


\section{Methods}

Coupled Lugiato Lefever equations. Interacting solitons can be described in optical fibre by using a coupled set of dynamical equations describing the slowly varying field amplitudes ${ }^{12,31}$. Here, these coupled equations have been adapted to a microresonator form consistent with the Lugiato Lefever (LL) equation ${ }^{7,32-34}$. By including cross-phase modulation and simplifying the Raman convolution terms under the assumption that the Raman response time is much faster than the soliton duration (see Supplementary Information for details), the following pair of equations results:

$$
\begin{gathered}
\frac{\partial E_{\mathrm{p}}}{\partial t}=i \frac{D_{2 \mathrm{p}}}{2} \frac{\partial^{2} E_{\mathrm{p}}}{\partial \phi^{2}}+i\left[g_{\mathrm{p}}\left|E_{\mathrm{p}}\right|^{2}+\left(2-f_{\mathrm{R}}\right) G_{\mathrm{p}}\left|E_{\mathrm{s}}\right|^{2}\right] E_{\mathrm{p}} \\
-i D_{1 \mathrm{p}} \tau_{\mathrm{R}} E_{\mathrm{p}} \frac{\partial\left(g_{\mathrm{p}}\left|E_{\mathrm{p}}\right|^{2}+G_{\mathrm{p}}\left|E_{\mathrm{s}}\right|^{2}\right)}{\partial \phi} \\
-\left(\frac{\kappa_{\mathrm{p}}}{2}+i \Delta \omega_{\mathrm{p}}\right) E_{\mathrm{p}}-\frac{\omega_{\mathrm{p}}}{\omega_{\mathrm{s}}} R\left|E_{\mathrm{s}}\right|^{2} E_{\mathrm{p}}+\sqrt{\kappa_{\mathrm{p}}^{\text {ext }} P_{\mathrm{in}}} \\
\frac{\partial E_{\mathrm{s}}}{\partial t}=-\delta \frac{\partial E_{\mathrm{s}}}{\partial \phi}+i \frac{D_{2 \mathrm{~s}}}{2} \frac{\partial^{2} E_{\mathrm{s}}}{\partial \phi^{2}}+i\left[g_{\mathrm{s}}\left|E_{\mathrm{s}}\right|^{2}+\left(2-f_{\mathrm{R}}\right) G_{\mathrm{s}}\left|E_{\mathrm{p}}\right|^{2}\right] E_{\mathrm{s}} \\
-i D_{1 \mathrm{p}} \tau_{\mathrm{R}} E_{\mathrm{s}} \frac{\partial\left(g_{\mathrm{s}}\left|E_{\mathrm{s}}\right|^{2}+G_{\mathrm{s}}\left|E_{\mathrm{p}}\right|^{2}\right)}{\partial \phi} \\
-\left(\frac{\kappa_{\mathrm{s}}}{2}+i \Delta \omega_{\mathrm{s}}\right) E_{\mathrm{s}}+R\left|E_{\mathrm{p}}\right|^{2} E_{\mathrm{s}}
\end{gathered}
$$

The slowly varying fields $E_{j}$ (subscript $j=(\mathrm{p}, \mathrm{s})$ for primary or Stokes soliton) are normalized to optical energy. To second order in the mode number, the frequency of mode number $\mu$ in mode family $j=(p, s)$ is given by the Taylor expansion $\omega_{\mu j}=\omega_{0 j}+D_{1 j} \mu+1 / 2 D_{2 j} \mu^{2}$, where $\omega_{0 j}$ is the frequency of mode $\mu=0$ in mode family $j$, while $D_{1 j}$ and $D_{2 j}$ are the FSR and the second-order dispersion at $\mu=0$. Also, $\delta=D_{1 \mathrm{~s}}-D_{1 \mathrm{p}}$ is the FSR difference between primary and Stokes solitons at mode $\mu=0 . \kappa_{j}$ is the cavity loss rate and $\Delta \omega_{j}$ is the detuning of mode zero of the soliton spectrum relative to the cold cavity resonance. For the primary soliton, which is a DKS, the pump field is locked to one of the soliton spectral lines and this 'pump' line is taken as mode $\mu=0 . \tau_{\mathrm{R}}$ is the Raman shock time ${ }^{8,9}, \kappa_{\mathrm{p}}^{\text {ext }}$ is the external coupling coefficient and $P_{\text {in }}$ is the pump power. $g_{j}$ and $G_{j}$ are self- and cross-phase modulation coefficients, defined as

$$
g_{j}=\frac{n_{2} \omega_{j} D_{1 j}}{2 n \pi A_{j j}}, \quad G_{j}=\frac{n_{2} \omega_{j} D_{1 j}}{2 n \pi A_{\mathrm{ps}}}
$$

where the nonlinear mode area $A_{j k}$ is defined as ${ }^{31}$

$$
A_{j k}=\frac{\iint_{-\infty}^{\infty}\left|u_{j}(x, y)\right|^{2} \mathrm{~d} x \mathrm{~d} y \iint_{-\infty}^{\infty}\left|u_{k}(x, y)\right|^{2} \mathrm{~d} x \mathrm{~d} y}{\iint_{-\infty}^{\infty}\left|u_{j}(x, y)\right|^{2}\left|u_{k}(x, y)\right|^{2} \mathrm{~d} x \mathrm{~d} y}
$$

where $u_{j}$ is the transverse distribution of the mode. $f_{\mathrm{R}}=0.18$ is the Raman contribution parameter in silica. $R=c D_{1 \mathrm{p}} g_{\mathrm{R}}\left(\omega_{\mathrm{s}}, \omega_{\mathrm{p}}\right) / 4 n \pi A_{\mathrm{ps}}$, where $g_{\mathrm{R}}\left(\omega_{\mathrm{s}}, \omega_{\mathrm{p}}\right)$ is the Raman gain in silica. For solitons with a few $\mathrm{THz}$ bandwidth, other effects are negligible in the resonators of this work (for example, higher-order dispersion, the self-steepening effect and Raman-induced refractive index change ${ }^{31}$ ).

Phase-sensitive, four-wave-mixing terms have been omitted in equations (2) and (3). In principle, these terms could introduce locking of the Stokes and primary soliton fields (in addition to their repetition rates). However, for this to occur the underlying spatial mode families would need to feature mode frequencies that align reasonably well (both in FSR and offset frequency) within the same band. In this work, the mode frequencies associated with each soliton did not overlap.
The coupled equations are numerically studied using the split-step Fourier method (see Supplementary Information). Over 600 modes are included in the simulation for each soliton. Parameters are given below. Note that the detuning of the Stokes soliton determines the rotation frame, which can be set to zero during the simulation.

Calculation of threshold. The behaviour of the Stokes soliton system can be studied analytically beginning with the coupled LL equations. Near threshold the Stokes soliton is much weaker in power than the primary soliton. In this limit, the cross-phase modulation and cross-Raman interaction terms within the primary soliton equation can be neglected, while the self-phase modulation and self-Raman terms are neglected in the Stokes equation. The primary soliton is then governed by the standard uncoupled LL equation, which features an analytical solution of the field amplitude (hyperbolic secant form $)^{7}$. This solution is substituted into the Stokes soliton equation, which then takes a Schrödinger-like form containing a $\operatorname{sech}^{2}$ potential in addition to $\mathrm{sech}^{2}$ Raman gain. The specific solution to these two equations in the Stokes low power limit take the following forms:

$$
E_{\mathrm{p}} \sim \operatorname{sech} B \phi, \quad E_{\mathrm{s}} \sim \operatorname{sech}^{\gamma} B \phi
$$

where $B=\sqrt{2 \Delta \omega_{\mathrm{p}} / D_{2 \mathrm{p}}}$. The Stokes soliton solution is the general solution for a soliton trapped in the index well created by the $\operatorname{sech}^{2}$ primary soliton intensity, where the power $\gamma$ satisfies the equation,

$$
\gamma(\gamma+1)=2\left(2-f_{\mathrm{R}}\right) G_{\mathrm{s}} D_{2 \mathrm{p}} / g_{\mathrm{p}} D_{2 \mathrm{~s}}
$$

Once the peak power of the primary soliton reaches a point that provides sufficient Raman gain to overcome round-trip loss, the Stokes soliton will begin to oscillate. The threshold condition emerges as the steady state Stokes soliton power balance. This is readily derived from the Stokes soliton equation, and takes the form

$$
\int_{0}^{2 \pi} \partial_{t}\left|E_{\mathrm{s}}\right|^{2} \mathrm{~d} \phi=\int_{0}^{2 \pi} \mathrm{d} \phi\left(\kappa_{\mathrm{s}}-2 R\left|E_{\mathrm{p}}\right|^{2}\right)\left|E_{\mathrm{s}}\right|^{2}=0
$$

By substituting the solutions (equation (6)) into equation (8), the resulting threshold in primary soliton peak output power is found to be given by equation (1)

Parameters. The measured parameters are: $\kappa_{\mathrm{p}} / 2 \pi=917 \mathrm{kHz}, \kappa_{\mathrm{p}}^{\mathrm{ext}} / 2 \pi=245 \mathrm{kHz}$, $\lambda_{\mathrm{p}}=1,550 \mathrm{~nm}, D_{1 \mathrm{p}} / 2 \pi=22 \mathrm{GHz}$ and $D_{2 \mathrm{p}} / 2 \pi=16.1 \mathrm{kHz}$. For the Stokes soliton mode family $\kappa_{\mathrm{s}} / 2 \pi=3.0 \mathrm{MHz}$ is fitted, but is within $10 \%$ of the value measured near $1,550 \mathrm{~nm}$ and is used for the Stokes soliton loss rate in the $1,600 \mathrm{~nm}$ band. Calculated parameters (based on mode simulations) are: $D_{2 \mathrm{~s}} / 2 \pi=21.7 \mathrm{kHz}$, $A_{\mathrm{ss}}=69.8 \mu \mathrm{m}^{2}, A_{\mathrm{pp}}=39.7 \mu \mathrm{m}^{2}, A_{\mathrm{ps}}=120 \mu \mathrm{m}^{2}$, and $\delta=0$ when $\lambda_{\mathrm{s}}=1,627 \mathrm{~nm}$. Other constants are: $n=1.45, n_{2}=2.2 \times 10^{-20} \mathrm{~m}^{2} \mathrm{~W}^{-1}, g_{\mathrm{R}}=3.94 \times 10^{-14} \mathrm{~m} \mathrm{~W}^{-1}$, $\tau_{\mathrm{R}}=3.2$ fs. $\gamma=0.55$ is calculated and used in Fig. 4 .

Data availability. The data that support the plots within this paper and other findings of this study are available from the corresponding author upon reasonable request.

\section{References}

31. Agrawal, G. P. Nonlinear Fiber Optics (Academic, 2007).

32. Lugiato, L. A. \& Lefever, R. Spatial dissipative structures in passive optical systems. Phys. Rev. Lett. 58, 2209-2211 (1987).

33. Matsko, A. et al. Mode-locked Kerr frequency combs. Opt. Lett. 36, 2845-2847 (2011)

34. Chembo, Y. K. \& Menyuk, C. R. Spatiotemporal Lugiato-Lefever formalism for Kerr-comb generation in whispering-gallery-mode resonators. Phys. Rev. A 87, 053852 (2013) 